\title{
STRUCTURAL OPTIMIZATION
}

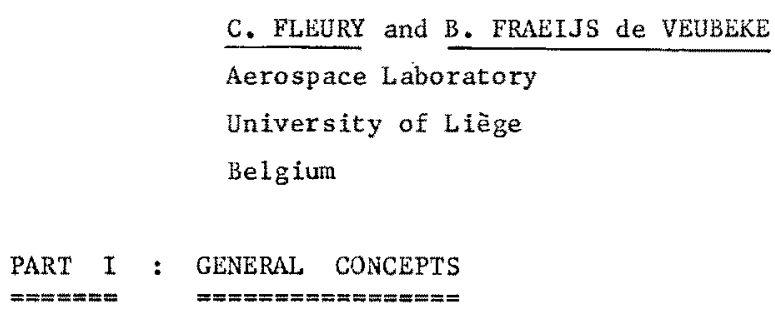

\section{INTRODUCTION}

Numerical methods of structural analys is have reached a high standard of efficiency. As a consequence they tend to overgrow their usefulness as numerical checks of stress distribution, amplitudes of displacement, natural frequencies and elastic stability to become adjuvants to design procedures.

The objectives of design vary according to the purpose of the structure. In aerospace engineering the weight is the prominent factor and is often the only goal of numerical optimization studies. In other cases the functional that is subject to minimization is more complex, economical factors of various $k$ ind being incorporated with their relative weights into the cost function. Until recently the minimization was carried out by trial and error, the preliminary design and the modifications introduced after evaluation of a numerical structural analys is being largely based on engineering judgment. Presently there is a tendancy to a more scientific approach in which the changes in design parameters are evaluated on the basis of algorithms. Efficient algorithms are those that tend to bring the functional to its minimum with the smallest number of iterations requiring a subsequent structural reanalysis. Moreover they have to satisfy many kinds od side constraints such as :

- remain within the elastic limits of the material in each structural member under a given set of load distributions;

- keep displacenent-type 1 initations;

- avoid elastic instability;

- keep natural frequencies within prescribed 1 in its;

- keep member sizes above minimum values.

The starting point of such automated Structural Optimization Programs is a given preliminary design. It is therefore difficult to evaluate the cost of optinization procedures, since the computer time devoted to reach a near-optimal stage will heavily depend on the quality of the preliminary design.

In case where the unicity of the optimal solution is not guaranteed a poor prelininary design can even lead to a local and not to the global minimum. 
For this reason, while optimization programs will probably remain essential tools, the objectives of optimality will also tend to incorporate the computer in the preliminary design stage. This more direct approach towards an optimal structure is the aim of "Computer aided Design".

It is also a much more ambitious goal and, fortunately perhaps, will never obliterate the exercize of engineering art. It is indeed difficult to conceive a selection by the computer of the best "topology" of structural members to carry the loads according to the purpose of the structure, taking irmediatly the effect of side constraints into account. On the other hand, once the topology has been fixed by engineering judgement and experience, we will probably reach the stage where the computer will carry out from there the sizing of the members and even such other alterations in their geometry, permissible under the given topology and external constraints. Whether such ambitious programs will ever become operational within economical 1 imits is a question that only experience will answer.

\section{DESIGN VARIABLES}

One can divide design variables in groups according to their relative importance. For aerospace structures, with a finite element method idealization, the following groups are proposed :

\subsection{Element sizes}

They comprize cross-sectional areas of beam, membrane and plate thicknesses ... The optimization of those variables alone leaves the topology (system of element interconnexions) and other geometrical characteristics (height, length, taper of beams, planforms of membranes and plates ...) unchanged.

\subsection{Geometric variables}

The choice of geometrical variables may alter the configuration of the structure but not its topology. In the finite element method they correspond to modifications in the nodal coordinates.

\subsection{Material properties}

The efficiency of the structure can be improved by a change of nature of the material selected for some of its members. For example Young's modulus and material density may be varied but this introduces discrete parameter modifications as opposed to the continuous variations possible in the previous design variables.

2.4. Topology

A change in topology is also, and more fundamentally so, a discrete modification to the structure. For example, a set of members may be replaced by a new one with different elements, differently connected.

The order in which the groups of design variables have been listed is roughly that of increasing complexity in an optimization program and attendant increasing cost. 
This consideration has led numerous research teams to 1 imit themselves to the first category. There is also some justification for it in the fact that the general layout of a structure is often dictated by other considerations than a certain definition of opt imality, Aerodynamic shape, headroom, access facilities, fallsafe design are characteristic examples.

The relative simplicity of dealing with element sizes only is enhanced by the choice of a finite element method for the discretization of the structure. As nodes are kept in place and element interconnexions are invariant, the statics and kinematics of the structure are not modified by alterations in element sizes. This can make a large part of the optimization program a fixed subroutine.

In the sequel we shall deal only with this restricted aspect of optimization.

\section{NUMERICAL METHODS OF STRUCTURAL OPTIMIZATION}

This section describes briefly two main approaches encountered in structural optimization and discusses their relative capabilities.

\subsection{Mathemat ical prograraming}

In this relatively recent approach, mininum weight design is treated as the mathematical problem of ext remizing a cost function in design space. Each dimension of this space is related to one design variable, so that each point corresponds to a possible design. The side-constraints consist of limits to the design variables (element sizes) themselves and to stresses or displacements, the latter constraints being generally functions of the design variables. Symbolically, denoting by $A_{i}(i=1 \ldots n)$ the design variables

$$
\begin{array}{ll}
W=W\left(A_{1} \ldots A_{n}\right) \text { min. } & \\
\underline{A}_{i} \leqslant A_{1} \leqslant \bar{A}_{i} & i=1 \ldots n \\
g_{j}\left(A_{1} \ldots A_{n}\right) \leqslant \bar{g}_{j} & j=1 \ldots P
\end{array}
$$

The cost function and the nature of the second type of constraints determine whether the problem can be treated by linear or non-linear programing. The second case usually prevails for structural optimization.

Drawbacks inherent to the mathematical programming approach appear with large numbers of design variables as the number of cycles required to get close to the optimum rapidly rises. Each cycle involves a costly stress reanalysis and the computational expenditure rapidly becomes prohibitive. On the other hand the method is very general and reliable. If a solution converges to a local minimum instead of the global minimum 
required, this can always be checked and the necessary steps be taken to reinitiate the procedure.

\subsection{Opt imality criteria}

Intuitive considerations as to the nature of the optimal design may lead to adopt optimality criteria that are not directly related to the minimization of the given cost function but sometimes constitute a satisfactory approximation to it. They can then provide a basis for the search techniques and lead to simple recursions formulas for redesign. The best known and widely used example of such a procedure is the "fully stressed design" concept. According to it, each component of an optimal structure is stressed to its $1 \mathrm{imit}$ in at least one of the loading conditions.

Convergence to the optimal solution, according to the fully stressed design criterion, is obtained in one iteration for statically determinate structures. In statically deterninate cases the internal loads are indeed independent of the design variables and opt mality based on fully stressed design coincides with the exact minimal weight criterion if no limitations are put on displacements. In the statically indeterninate case each redesign modifies the internal loading distribution and fully stressed design does not yield the minimum weight but may be considered to approach it satisfactory.

An attractive feature of fully stressed design that explains its relative success is its tendancy to converge in a number of cycles independent of the number of design variables, in contrast to the more rigorous mathematical programming method. Moreaver each redesign cycle is fairly simple.

\section{REFERENCES}

(1) L.A. SCHMIT and R. H. MALETT

"Structural Synthesis and Design Parameter Hierarchy" Journal of the Structures Division, ASCE, vol. 89, August 1963, p. 269.

(2) C.Y. SHEU and W. PRAGER

"Recent Developments in Optimal Structural Design" Applied Mechanics Review, 21, 1968, p. 985.

(3) R.A. GELLATLY

"The Role of Optimization in the Design of Aircraft Structures" Proceedings of AGARD Symposium on Structural Optimization, Ist ambu1, AGARD-CP-36-70, 1970, paper 9.

(4) F.I. NIORDSON and P. PEDERSEN

"A Review of Optimal Structural Design"

DCAMM Rep. 31, Dept. of Solid Mechanics, The Technical University of Denmark, Copenhagen, 1972. 
PART IT : AN ALGORTTHM FOR MINIMUM WEIGHT DESIGN UNDER A

SET OF LOADING MODES WITH CONSTRAINTS ON STRESSES

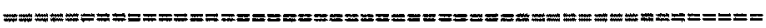

AND DISPLACEMENTS.

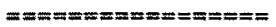

\section{PROBLEM DEFINITION}

The structure is in the linear elastic regime and idealized by finite elements. Under all the specified loading distributions certain constraints on stresses and nodal displacements must be satisfied. The geometry and the material properties are predetermined.

The functional to be minimized

$$
W=\sum_{i} \rho_{i} L_{i} A_{i} \quad i=1 \ldots n_{e}
$$

is the structural weight, proportional in each element to the material density $p_{i}$, to the design variable $A_{i}$ (cross-section of bar , thickness of membrane,...) and a geometrical parameter $L_{i}$ (length of bar, area of membrane...).

\section{DESCRIPTION OF THE CONSTRAINTS}

\subsection{Production constraints}

They place a lower and sometimes an upper $1 \mathrm{mit}$ to the design variables:

$$
A_{i} \leqslant A_{i} \leqslant \bar{A}_{i}
$$

\subsection{Stress 1 imitations}

In bar-type elements the tensile stress linit is determined by elastic the $v_{\text {properties }}$ of the material; the compressive 1 imit may be reduced to take into consideration, in a simple manner, a safeguard against buckling. If $\sigma_{i}$ is the actual stress in the bar: 


$$
\underline{\sigma_{i}} \leqslant \sigma_{i} \leqslant \overline{\sigma_{i}}
$$

In shear panels one assumes a maximum allowable shear stress, usually governed by buckling considerations :

$$
\tau_{i} \leqslant \overline{\tau_{i}}
$$

In more general membrane elements, where the three stress conponents $\sigma_{x}, \sigma_{y}$ and $\tau_{x y}$ play equally important roles, a reference stress related to an elastic limit criterion may be introduced.

\subsection{Displacement constraints}

They assign upper bounds to generalized displacements. To determine analytical expressions for them in terms of the design variables, the virtual work theorem is used.

If $F$ denotes a vector (column matrix) of externaliy applied loads,

$u$ the conjugate vector of generalized displacements,

o the stress vector,

$\varepsilon$ the conjugate strain vector,

the virtual work is given by :

$$
\Delta=F_{(v)}^{T} u_{(r)}=\int_{V} \sigma_{(r)}^{T} \varepsilon_{(v)} d V=\int_{V} \sigma_{(v)}^{T} \varepsilon_{(r)} d V
$$

The subscripts between brackets refer to either a virtual or a real vector, the superscript $T$ denotes transposition. Splitting the integral into the sum of contributions of each finite element:

$$
\Delta=\Sigma \int_{V_{i}}\left(\sigma^{T}(r) E(v)\right)_{i} d_{i}
$$

According to the finite element theory we have:

$$
\int_{V_{i}}\left(\sigma_{(r)}^{T} \varepsilon_{(v)}\right)_{i} d v_{i}=q_{(r) i}^{T} k_{i} q_{(v) i}=q_{(r) i}^{T} g(v) i
$$

where $q_{i}$ is the vector of generalized displacements of element $i$ and $g_{i}$ its conjugate of generalized loads. $K_{i}$ is the stiffness matrix of the element.

Let now $u_{j}$ denote a displacement component of the nodal displacement vector of the structure. Applying a corresponding virtual unit load to the structure, (7) and (8) give :

$$
u_{j}=\sum_{i} q_{(r) i}^{T} g_{i(j)}
$$


where the $g_{i(j)}$ are the corresponding virtual loads generated at each element level. In statically determinate structures the loads $g_{\hat{i}(j)}$ are uniquely determined by the unit load and (9) turns out to be given in terms of the design variables by:

$$
u_{j}=\sum_{i} \frac{c_{i j}}{A_{i}}
$$

where the $c_{i j}$ are constants. In redundant structures those coefficients are themselves implicit functions of the design variables.

\section{FORMULATION}

Let us begin with the statically determinate case

\subsection{Analys is stage}

The structure is analyzed under

- the $n_{r}$ real loading systems of the design specification

- the $n_{v}$ virtual loading cases connected with each displacement constraint.

\subsection{Redesign stage}

If the stress constraints are:

$$
\begin{aligned}
\sigma_{i \ell} \leqslant \bar{\sigma}_{1 \ell} & i=1 \ldots n_{e} \\
\ell & =1 \ldots n_{r}
\end{aligned}
$$

where $\sigma_{i \ell}$ is the actual stress in element $i$ under the loading case $l$, the redesign is effected in a single step by:

$$
A_{i}^{*}=A_{i} \quad \max \left\{\frac{\sigma_{i \ell}}{\bar{\sigma}_{i \ell}}\right\}
$$

with, in addition, the minimum size requirement:

$$
A_{\dot{i}}^{*} \geq \underline{A_{i}}
$$

This method provides a "fully stressed" design, each element reaching its limiting stress (or having its minimal size) under at least one of the loading cases. If we have displacement constraints, the analysis stage will provide the matrix of $c_{i j}$ coefficients appearing in (10). Taking the $A_{i}^{*}$ values appearing in (12) as minimal, the problem with the addition of displacement constraints can be stated as follows: 
under

$$
W=\sum_{i} p_{i} L_{i} A_{i} \quad \text { min }
$$

$$
\begin{aligned}
& \sum_{i} \frac{c_{i j}}{A_{i}} \leqslant \bar{u}_{j} j=1 \ldots n_{t} \\
& \bar{A}_{i} \geqslant A_{i} \geqslant A_{i}^{*} \quad i=1 \ldots n_{e}
\end{aligned}
$$

where

$$
\mathrm{n}_{\mathrm{t}}=\mathrm{n}_{\mathrm{r}} \times \mathrm{n}_{\mathrm{v}} \quad \text {. }
$$

Because of the assumption of statical determinacy the formulation is rigorous, the solution unique and only one stress analysis is required.

Real structures, however, are rarely statically determinate. If subjected to different load distributions they are in fact both stiffer and even lighter if proper use is made of the stress cooperation provided by redundancy. But in this case both the $c_{i j}$ and the $A_{i}^{*}$ become implicit functions of the design variables. Each change in those will produce new $c_{i j}$ and $A_{i}^{*}$ that can only be known exactly through a costly stress reanalysis. The following approach is suggested. The problem as defined by equations (14) and (15) is solved by considering the $c_{i j}$ and $A_{i}^{*}$ as constants. The evolved solution for the design variables is inserted in a new stress analys is to provide new $c_{i j}$ and $A_{i}^{*}$ values with which to reinitiate problem (14), (15) unti11 close to convergence.

\section{SOLUTION OF THE LINEARIZED PROBLEM (14), (15)}

In order to solve this problem, in which the $c_{i j}$ and $A_{i}^{*}$ are assumed to be given, it is beneficial to take the reciprocals of sizing variables as new design variables. The recast problem may then be solved by means of the gradient projection method for linear constraints (ref. [6]) adapted to the problem under consideration. As required by this method, the fnitial point must be a feasible point, a point lying in the convex region formed by the prescribed constraints. In any given case, such a point can readily be found by linear scaling of all member sizes, so that a feasible bounded design is generated (one constraint at its critical value, others subcritical). This scaling of all the design variables does not introduce stress redistribution : each stress and each displacenent are simply divided by the same scaling factor.

\section{APPLICATION OF THE METHOD}

Ailongst known optimization programs we can mention :

- GELLATLY and BERKE (ref. [2] )

- TAIG and KERR (ref. [3]). 
As mentioned previously a structural optimization program performs iterative cycling between a structural analysis stage and a redesign stage. The programs under study at the Aerospace Laboratory of the University of Liège are coupled to the extensively developped ASEF code as the analysis module. The structural idealization consists up to now of axial force members and triangular or quadrilateral membrane elements. The degree of displacement polynomials within the elements is allowed to vary from 1 to 3 . A symmetry option has been introduced, that constrains members of any specifled group to be identical; in this case, the number of design variables is reduced to the number of element groups.

6. EXAMPLES

The method proposed in section 3 has been tested against solutions to classical problems found in the literature.

The two first examples show clearly that the rate of convergence of the redesign procedure is not directly related to the size of the problem under consideration.

\subsection{Four-Bar Pyramid (fig. 1)}

This very simple structure is subjected to the single loading case given fig.l. Constraints are placed on maximum stress (25000 psi), minimum area ( $0.1 \mathrm{in}_{*}^{2}$ ) and node displacenent in $z$-direction $(0.3 \mathrm{in.}$.). The present results (table I) duplicate those of ref. [2] and [3]. Fig, 4.a shows the strange pattern followed by the iteration procedure : the design seems to converge after 2 cycles and only after several more cycles does the rate of weight reduction accelerate till the final design is generated (after 20 iterations).

\subsection{2- Bar Four Level Tower (fig. 3)}

This doubly symetric tower is subjected to two loading cases (table II-a). Symmetry is achieved by use of the input option, which reduces the number of design variables from 72 to 16 . The stress limits are again $25000 \mathrm{psi}$. with 0.1 in $^{2}$. minimum area. The displacenents of the four uppermost nodes are limited to $0.25 \mathrm{in}$. in the $x$ and $y$-directions. In spite of the larger size of the problem, convergence is very rapid and optimal design is reached in only five iterations (see fig. 4.b). The results (table II-b) are the same as those of ref. $[3]$.

\subsection{Cantilever Frame (fig.2)}

The 10 barmtruss is subjected to the single loading case indicated on $\mathrm{fig} .2$. The stress limit in all members is $25000 \mathrm{psi}$. with $0.1 \mathrm{in}^{2}$, minimum area. The node 
displacements in y-direction are prescribed to be less than 0.2 in. Table III shows the results obtained from the present method and, for comparison, from other methods (ref. $[1],[2],[3]$ ).

In addition, stress constraints have been formulated in a similar way than for displacement constraints:each member stress is 1ijeariy expressed in terms of the inverses of the design variables. Corresponding results are given in table III under the title "Experimental Method". This method generates a design weighting 5060.8 1b. which exhibits the following particular characteristics. Member 6 is fully stressed, while being at its minimum area. Furthemore only one displacenent constraint is exactly satisfied (node 1) while another prescribed displacement is close to its limiting value (node 3 ).

For the other designs, shown on table III, these two displacements reach simultaneously their liniting values.

\section{CONCLUSION}

While using a mathematical programing algorithm, the method that was presented has the convergence characteristics of an optimality critexion approach. Except for the last example (cantilever frame), the same results as those of Taig and Kerr (ref. [3]) have been obtained for each analysis and redesign step. In addition, when there is only one active displacement constraint, the results of Gellat ly and Berke (ref. [2]) are also identical to ours. In fact, all these methods are based on the same technique : by means of the virtual work theorem each linited displacement (or 1 inear combination of displacements) is expressed in terms of the design variables. The resulting relations renain exact only in the case of a statically determinate structure; for a redundant structure, they become approximated. The redesign procedures are characterized by the algorithn used in order to resolve the ensuing linearized problem (14) (15).

The present method has the advantage of using a particularly suggestive algorithm : each path up to an "approached" optimum readily shows whether a constraint becomes active or not.

Furthermore each point of this path is a feasible bounded point. That important feature allows the algorithm to be eventually stopped before reaching the optimum, in order to avoid a final divergence due to a too strong internal redundancy. 


\section{REFERENCES}

(1) V, B, VENKAYYA

"Design of Optimum Structures"

Computers and Structures, vol. 1, Nos 1/2, Aug. 1971, p. 265.

(2) R. A. GELLATLY and L. BERKE

"Optimal Structural Design"

USAF Technical Report AFFDL-TR-70-165, February 1971.

(3) I. C. TAIG and R. I. KERR

"Optimization of Aircraft Structures with Multiples Stiffness Requirements" Second Symposium on Structural Optimization, AGARD CONFERENCE PROCEEDINGS $n^{\circ} 123$, paper 16.

(4) J. M. CHERN and W. PRAGER

"Opt Imal Design of Beams for Prescribed Compliance under Alternative Loads" Journal of Optimization Theory and Application, vol. 5, $\mathrm{n}^{\circ} 6,1970$, p. 424.

(5) J.M. CHERN and W. PRAGER

"Minimum weight Design of Statically Deteminate Trusses Subjected to Multiples Constraint s".

International Journal of Solids and Structures, vol.7, 1971, p. 931.

(6) J. B. ROSEN

"The Gradient Projection Method for Nonlinear Programing.

Part I. Linear Constraints".

Journal of the Society for Industrial and Applied Mathematics, 8, 1960, p.181.

(7) C. FLEURY

"Methodes numériques d'optimisation des structures"

Rapport SF-19, Laboratoire de Techniques Aéronautiques et Spatiales, Liège, Belgium.

(8) C. FLEURY

"Applications pratiques des mëthodes numériques d'optimisation structurale " Rapport SF-28, Laboratoire de Techniques Aéronautiques et Spatiales,

Liège, Belgium. 


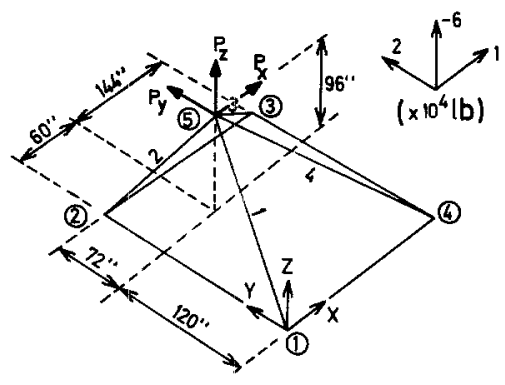

FOUR BAR PYRAMID

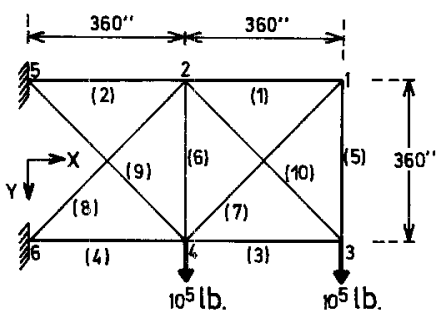

CANTILEVER FRAME

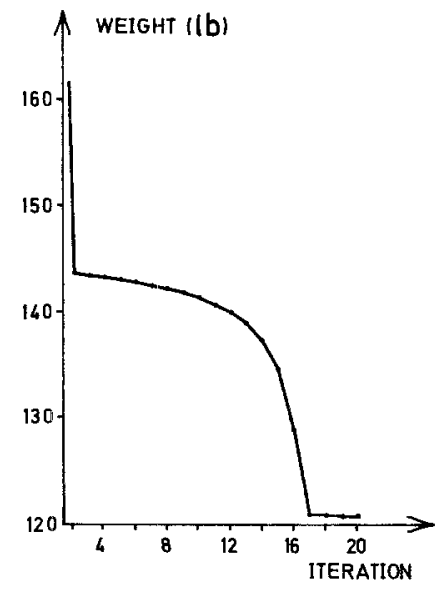

(a) four-bar pyramid
FIG. 1

FIG. 2

FIG. 3

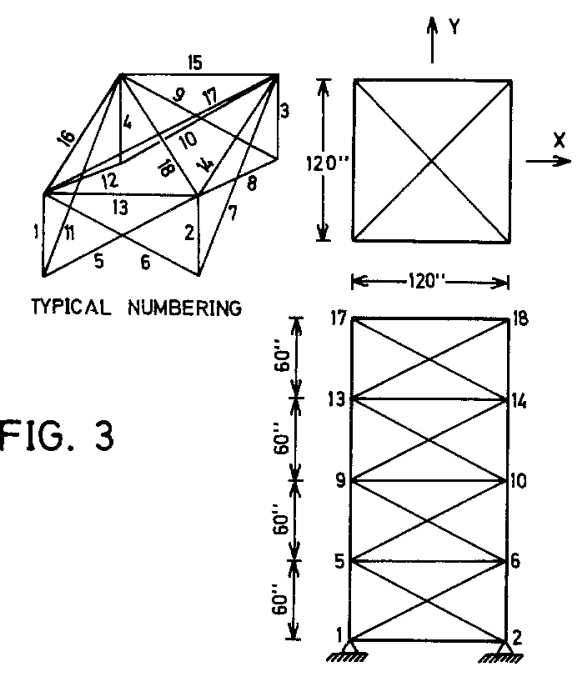

72 - BAR TOWER
FIG. 4

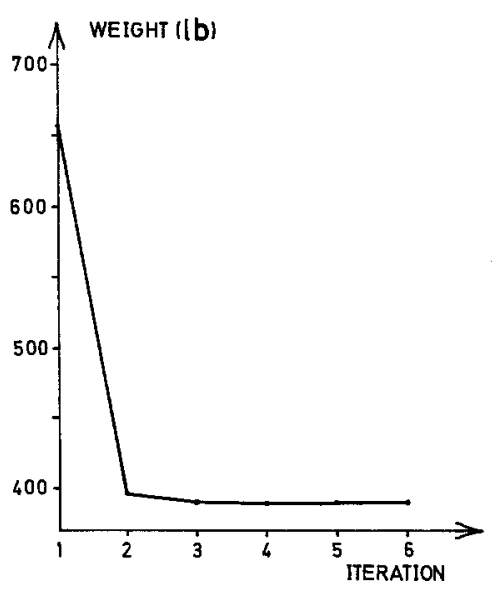

(b) 72-bar tower 
TABLE I FOUR BAR PYRAMTD

\begin{tabular}{|c|c|c|c|c|c|}
\hline \multirow{2}{*}{ WEIGHT } & \multirow{2}{*}{$\begin{array}{l}\mathrm{NO}_{\mathrm{O}} \text { OF } \\
\text { ITERATION }\end{array}$} & \multicolumn{4}{|c|}{ MEMBER SIZES } \\
\hline & & 1 & 2 & 3 & 4 \\
\hline 120.73 & 22 & 0.100 & 3.893 & 0.747 & 2.510 \\
\hline
\end{tabular}

TABLE II 72-BAR TOWER

(a) Loading systems (1b)

(b) Final Design

\begin{tabular}{|c|c|c|c|c|}
\hline $\begin{array}{l}\text { Load } \\
\text { case }\end{array}$ & Node & $X$ & $Y$ & $Z$ \\
\hline 1 & 17 & 5000 & 5000 & -5000 \\
\hline 2 & 17 & 0 & 0 & -5000 \\
& 18 & 0 & 0 & -5000 \\
& 19 & 0 & 0 & -5000 \\
& 20 & 0 & 0 & -5000 \\
\hline
\end{tabular}

Final Weight : $379,66 \mathrm{lb}$

No of iteration : 5

\begin{tabular}{|c|c|c|c|}
\hline \multicolumn{2}{|c|}{ Meruber Size } & \multicolumn{2}{|c|}{ Member size } \\
\hline $1-4$ & 1.897 & $37-40$ & 0.507 \\
$5-12$ & 0.516 & $41-48$ & 0.520 \\
$13-16$ & 0.100 & $49-52$ & 0.100 \\
17,18 & 0.100 & 53,54 & 0.100 \\
$22-12$ & 1.280 & $55-58$ & 0.157 \\
$23-30$ & 0.515 & $59-66$ & 0.536 \\
$31-34$ & 0.100 & $67-70$ & 0.410 \\
35,36 & 0.100 & 71,72 & 0.654 \\
\hline
\end{tabular}

TABLE III CANTILEVER FRAME

\begin{tabular}{|c|c|c|c|c|c|}
\hline $\begin{array}{l}\text { MEMBER } \\
\mathrm{N}_{\mathrm{O}}\end{array}$ & $\operatorname{REF} \cdot[3]$ & $\mathrm{REF} \cdot[2]$ & $\operatorname{REF} \cdot[1]$ & $\begin{array}{l}\text { PRESENT } \\
\text { METHOD } \\
(\mathrm{x})\end{array}$ & $\begin{array}{l}\text { EXP. } \\
\text { METHOD }\end{array}$ \\
\hline $\begin{array}{r}1 \\
2 \\
3 \\
4 \\
5 \\
6 \\
7 \\
8 \\
9 \\
10\end{array}$ & $\begin{array}{r}0.10 \\
31.98 \\
15.43 \\
22.57 \\
0.57 \\
0.58 \\
0.10 \\
22.76 \\
21.82 \\
6.44\end{array}$ & $\begin{array}{r}0.10 \\
31.35 \\
15.60 \\
20.03 \\
0.24 \\
0.14 \\
0.10 \\
22.21 \\
22.06 \\
8.35\end{array}$ & $\begin{array}{r}0.13 \\
30.42 \\
14.90 \\
23.41 \\
0.10 \\
0.10 \\
0.19 \\
21.08 \\
21.08 \\
8.70\end{array}$ & $\begin{array}{r}0.10 \\
30.73 \\
14.73 \\
23.94 \\
0.10 \\
0.10 \\
0.10 \\
20.95 \\
20.84 \\
8.54\end{array}$ & $\begin{array}{r}0.10 \\
30.52 \\
15.22 \\
23.20 \\
0.55 \\
0.10 \\
0.10 \\
21.03 \\
21.53 \\
7.46\end{array}$ \\
\hline $\begin{array}{l}\text { TOTAL } \\
\text { WEIGHT }\end{array}$ & 5167 & 5112.17 & 5084.90 & 5076.67 & 5060.85 \\
\hline $\begin{array}{l}\text { No.OF } \\
\text { ITERATIONS }\end{array}$ & 32 & 19 & 25 & 14 & 19 \\
\hline
\end{tabular}

(x) Taig and kerr have also obtained this result by fixing member 5 at its minimum area. 